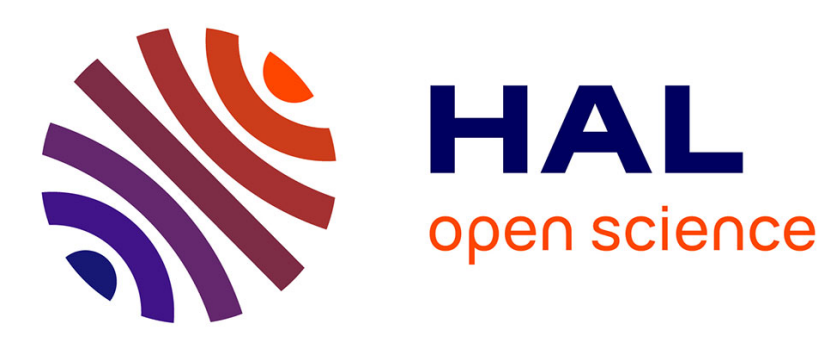

\title{
L'idée de point de vue sociologique
}

Michel Bourdeau

\section{To cite this version:}

Michel Bourdeau. L'idée de point de vue sociologique: la philosophie des sciences comme sociologie des sciences chez Auguste Comte. Cahiers Internationaux de Sociologie, 2004, 1 (1), pp.225-238. halshs-00855933

\section{HAL Id: halshs-00855933 \\ https://shs.hal.science/halshs-00855933}

Submitted on 30 Aug 2013

HAL is a multi-disciplinary open access archive for the deposit and dissemination of scientific research documents, whether they are published or not. The documents may come from teaching and research institutions in France or abroad, or from public or private research centers.
L'archive ouverte pluridisciplinaire HAL, est destinée au dépôt et à la diffusion de documents scientifiques de niveau recherche, publiés ou non, émanant des établissements d'enseignement et de recherche français ou étrangers, des laboratoires publics ou privés. 
Michel Bourdeau: Cahiers internationaux de sociologie, 1 (2004) 225-238, L'idée de point de vue sociologique

\title{
L'IDÉE DE POINT DE VUE SOCIOLOGIQUE.
}

\section{LA PHILOSOPHIE DES SCIENCES COMME SOCIOLOGIE DES SCIENCES CHEZ AUGUSTE COMTE.}

\author{
MICHEL BOURDEAU, \\ (CAMS-CNRS) \\ bourdeau@ehess.fr
}

\begin{abstract}
"le point de vue sociologique est désormais, en tous genres, le seul vraiment philosophique" ${ }^{1}$
\end{abstract}

Pour qui aborde Comte pour la première fois, la première difficulté est de comprendre de quel lieu parle ce polytechnicien qui fut l'une des têtes les plus fortes de son temps. Si l'on entend par scientisme l'admiration béate de la science, il doit être clair par exemple qu'il fut tout sauf scientiste, et cela dès le début. Certes, la science est valorisée. Les termes qui servent à qualifier chacun des trois états ne sont pas mis sur le même plan. Théologie et métaphysique sont données pour disparues, ou destinées à disparaître, alors que la science, elle, représente l'état normal et définitif de l'intelligence humaine. Il n'y a pas de quatrième état, et l'erreur de la théologie et de la métaphysique consiste précisément à croire qu'il existerait des formes de connaissance supérieure à la connaissance scientifique. Pour voir dans le positivisme un scientisme, il faudrait oublier que la science y est jugée, et parfois très sévèrement. Comte, qui connaissait le monde scientifique de l'intérieur, a perdu rapidement toute illusion à son sujet. La science enfle et dessèche; la spécialisation qui y règne

\footnotetext{
${ }^{1}$ C, $58^{\text {ième }}$ leçon, p. 100.

Pour les oeuvres de Comte, nous citerons le Cours (abrégé en : C) dans l'édition Hermann (2 volumes, Paris, 1975), en indiquant le numéro de la leçon, puis la page, sans mention du volume, étant entendu que le second contient les leçons 46 à 60.

Pour les leçons 47 à 51, les références renvoient aux Leçons de sociologie, Paris, GF, 1995. Pour les leçons 58 à 60, les références renvoient à Science et politique, les Conclusions générales du Cours de philosophie positive, Paris, Pocket, 2003.

Le Discours sur l'esprit positif (abrégé en : 1844) est cité dans l'édition donnée par A. Petit, Paris, Vrin, 1993.

Le Discours sur l'ensemble du positivisme (abrégé en : 1848), est cité dans la réédition qu'en a donnée A. Petit, GF, 1998.

Le Système (abrégé en $S$, suivi du numéro de tome) est cité dans la réédition procurée par la Société Positiviste, Paris, 1927, quatre volumes.
} 
engendre une indifférence générale au cours du monde. A la différence de bien de ses contemporains, on chercherait en vain chez le fondateur de la religion de l'humanité une quelconque religion de la science; au contraire, si la nouvelle religion transforme les lois scientifiques en dogme, ce n'est que pour subordonner le dogme au culte.

Pour caractériser d'un mot le lieu d'où parle Comte, le moins inapproprié est peut-être encore de dire qu'il raisonne en sociologue, à condition toutefois d'ajouter aussitôt que l'idée que se faisait de la sociologie celui qui en a forgé le nom est très éloignée de celle qui a cours aujourd'hui. De même que Russell avait proposé d'adopter un point de vue logique, de même Comte nous propose d'adopter un point de vue sociologique. Mais que faut-il entendre au juste par là?

La réponse procédera en trois temps. Le point de vue sociologique, en première approximation, n'est rien d'autre que le point de vue philosophique. Décrivant l'enchaînement de ses deux carrières, Comte déclarait avoir, dans la première, transformé la science en philosophie avant de transformer, dans la seconde, celle-ci en religion. Or le Cours marche inexorablement vers la fondation de la sociologie, et c'est donc avec la création de ce qui était alors la science finale que la distinction entre science et philosophie se trouve abolie. Reste alors à savoir pourquoi introduire un nouveau terme, si l'on pouvait tout aussi bien continuer à employer l'ancien. Le mot sociologie est chargé d'indiquer la place centrale occupée, dans la pensée positive, par la question des rapports de la science et de la société, la prépondérance du point de vue social entraînant en particulier un droit de regard de la raison publique sur l'activité scientifique. Enfin, le point de vue sociologique rend possible l'apparition de cette méthode subjective suivie par Comte dans le Système et que l'on peut tenir pour largement responsable des errements de sa seconde carrière. Ramenée à ses justes dimensions, ce n'est pourtant qu'un corollaire de la prépondérance accordée au point de vue humain, prépondérance qui, en principe, ne remet pas en cause les prérogatives reconnues à la méthode objective.

\section{La sociologie, entre science et philosophie.}

Avec le recul du temps il est clair que Comte appartient bien davantage à l'histoire de la philosophie qu'à celle de la sociologie. Pour comprendre le point de vue auquel il a choisi de se placer, le plus simple est donc de dire qu'il parle en philosophe. Mais il fait du mot un usage bien à lui et qui a varié au cours du temps. Ainsi, ce n'est que rétrospectivement qu'il s'est flatté d'avoir, dans le Cours, transformé la science en philosophie et les écrits de jeunesse proposent une autre vision des rapports de la science à la philosophie, qu'ils s'attachent plutôt à distinguer ${ }^{2}$. En particulier, l'ancien secrétaire de Saint-Simon semble avoir été le premier à dégager explicitement le concept de philosophie d'une science, ce qui fait de lui le premier épistémologue moderne ${ }^{3}$. La première leçon

\footnotetext{
${ }^{2}$ A. Petit: Des sciences positives à la politique positiviste, in A. Petit (éd.) : Auguste Comte, trajectoires positivistes, Paris, L'Harmattan, 2003, p. 87-117.

${ }^{3}$ Notamment dans une lettre à Valat, où il déclare qu'une épistémologie internaliste est seule en mesure de nous donner des règles efficaces pour la direction de l'esprit : “Ce n'est point a priori, dans sa nature, que l'on peut étudier l'esprit humain et prescrire des règles à ses opérations; c'est uniquement a posteriori, c'est-à-dire d'après ses résultats, par des observations sur des faits, qui sont des sciences. C'est uniquement par des observations bien faites sur la manière générale de procéder dans chaque science, sur les différentes marches que l'on y suit pour procéder aux découvertes, sur les méthodes en un mot, que l'on peut s'élever à des règles sûres et utiles sur la manière
} 
du Cours est on ne peut plus explicite : l'auteur y poursuit deux buts, un but spécial, la constitution de ce qui ne s'appelle pas encore sociologie mais physique sociale, un but général, la systématisation des sciences, étant bien entendu que ces deux objets, "quoique distincts en eux-mêmes, sont nécessairement inséparables". Quand l'auteur ajoute : "En un mot, c'est un Cours de philosophie positive, non de sciences positives, que je me propose de faire $^{4 \prime \prime}$, cela se rapporte bien sûr au second but. Mais il existe encore un autre usage, où la philosophie n'est plus pensée dans son rapport aux sciences. C'est celui que l'on trouve par exemple dans l'exposé de la loi des trois états, qui associe à ces derniers trois sortes de philosophies, définies cette fois comme "systèmes généraux de conceptions sur l'ensemble des phénomènes". Les deux usages ont en commun l'accent mis sur la systématicité et sur la généralité. Pour les articuler, pour penser "la destination de la philosophie positive dans le système général des sciences positives proprement dites", Comte proposera "de faire de l'étude des généralités scientifiques une grande spécialité de plus" ${ }^{2}$. Les sociologues auxquels il croyait pouvoir confier cette tache ont décliné, comme on sait, l'invitation et aujourd'hui encore on ne voit à peu près personne, hormis les philosophes, qui remplisse tant bien que mal les fonctions décrites par l'ancien polytechnicien.

Latente en 1830 dans les préliminaires du Cours, la tension entre science et philosophie s'accentue et devient manifeste en 1842, dans ses conclusions générales. Entre ces deux dates en effet, un événement décisif s'est produit, la naissance de la sociologie. Pour se convaincre du statut singulier de celle-ci, il suffit de noter son caractère totalisateur, qui lui permet de tout englober, notamment les cinq sciences qui la précèdent. Les épistémologues qui étudient le Cours se dispensent d'ordinaire de lire les trois derniers tomes, estimant que les leçons de sociologie ne sont pas de leur ressort. S'il est vrai que, de leur point de vue, le plus intéressant se trouve dans les leçons que les trois premiers volumes consacrent à chaque science en particulier, il n'en reste pas moins que la dynamique sociale revient, de façon certes beaucoup moins approfondie, sur les grands moments de l'histoire des sciences. L'épistémologie comtienne a donc ceci de tout à fait remarquable d'examiner chaque science deux fois, et donc, pour utiliser le jargon contemporain, d'adopter tour à tour les points de vue internaliste et externaliste donnés le plus souvent pour incompatibles. En ce sens, la sociologie possède un double statut : elle n'est pas seulement la science des faits sociaux; elle accomplit cette systématisation du savoir annoncée dans la première leçon et se place à ce titre sur un autre plan que les sciences qui la précèdent ${ }^{6}$.

de diriger son esprit. Ces règles, ces méthodes, ces artifices composent dans chaque science ce que j'appelle sa philosophie. Si l'on avait des observations de ce genre sur chacune des sciences reconnues comme positives, en prenant ce qu'il y aurait de commun dans toutes les résultats scientifiques partiels, on aurait la philosophie générale de toutes les sciences " A Valat, 24 sept. 1819; Correpondance Générale, Paris, Mouton puis Vrin, 1973-1990, t. 1, p. 59

${ }^{4}$ C., $1{ }^{\text {ère }} 1$., respectivement p. 29 et 30.

${ }^{5}$ C., $1{ }^{\text {ière }} 1$., respectivement, p. 2132 et 31.

${ }^{6}$ La situation a été fort bien décrite par Jean Lacroix : "La sociologie et la philosophie coïncident. Aussi ce terme de sociologie ne va-t-il pas sans ambiguïté chez Auguste Comte : il désigne tantôt une science particulière, celle des faits sociaux, tantôt l'ultime systématisation du savoir, c'est-à-dire la philosophie même - tantôt une science objective comme toutes les autres, tantôt une théorie de la connaissance subjective. Et c'est peut être cette ambiguïté radicale [...] qui fait à la fois toute la faiblesse 
Les conclusions générales du Cours ont pour fonction de prendre acte des modifications introduites et d'en dégager la portée. Comte s'y montre conscient de son originalité. Il s'y félicite d'avoir "réalisé un nouveau mode philosophique" et la dernière page de la cinquante huitième leçon, présentée comme l'équivalent du Discours de la méthode, revient encore sur le mouvement qui "élève peu à peu l'esprit scientifique proprement dit à la dignité finale d'esprit vraiment philosophique, en dissipant à jamais la distinction provisoire qui devait subsister entre eux tant que l'évolution préliminaire du génie moderne n'était pas suffisamment opérée" ${ }^{\prime 7}$. Brunschvicg, qui avait très bien saisi l'importance de telles déclarations, accusait leur auteur d'avoir, en associant le génie philosophique à la généralité et le génie scientifique à la positivité, commis un lapsus révélateur et abandonné de facto le positivisme de la positivité ${ }^{8}$. A ses yeux d'ailleurs, le mal était fait depuis longtemps, depuis le moment où abordant la biologie, Comte avait cru bon de changer de méthode et de substituer la synthèse à l'analyse. Dans la volonté affichée de maintenir l'équilibre entre les demandes respectives de la science et de la philosophie, de la positivité et de la généralité, il ne fallait voir en réalité qu'un subterfuge destiné à dissimuler l'impérialisme sociologique. Une chose est sûre toutefois. Quelques mois avant sa mort le grand prêtre de l'Humanité proposait de distinguer, au sein de l'état positif, deux modes successifs, scientifique puis philosophique, le second seul étant définitif. La science se révélait "aussi préliminaire que la théologie et la métaphysique" et se voyait même refuser "l'attribut de pleine positivité" ${ }^{9}$.

Le pouvoir unificateur de la science sociale prend encore un autre aspect. Le premier essor de la pensée scientidique en Grèce avait vite amené à distinguer entre philosophie naturelle et philosophie morale; "fatal antagonisme" qui depuis lors avait commandé la marche de l'esprit humain, et que la sociologie vient également abolir puisqu'avec elle la science, jusque là cantonnée à l'étude des phénomènes naturels, prend possession des phénomènes moraux que la théologie continuait à revendiquer comme siens. Sans entrer dans la question des rapports du positivisme et de la morale, il importe de préciser que, dans l'usage que celui-ci fait du terme, l'accent n'est pas mis sur la normativité. L'étymologie rattache la morale aux moeurs, et l'on pensera également à la façon dont Cabanis opposait le physique au moral. Le partage établi en Grèce excluait la morale du champ de la science et, deux mille ans plus tard,

sociologique et toute la grandeur philosophique du positivisme. En tout cas, l'avènement de la philosophie positive est, du même mouvement, une promotion de l'esprit scientifique et une promotion de l'esprit philosophique. C'est la subordination de toutes les sciences à la sociologie qui réintroduit l'idée de philosophie dans le positivisme et l'éloigne du scientisme. La science devient philosophie en se réorganisant du dedans en vue de son utilité publique" (La sociologie d'Auguste Comte, P.U.F, 1961, p. 11-12). On trouverait des développements parallèles dans le chapitre qu'Alain a consacrées à Comte dans Idées.

${ }^{7}$ C., $58^{\text {ième }} 1$., respectivement p. 73 et 158 . Par un tout autre chemin, à savoir le rejet de la distinction entre analytique et synthétique, Quine était arrivé à une position qui n'est pas sans analogie avec celle-ci. Pour le philosophe de Harvard, il est des cas où la frontière entre science et philosophie est difficile à tracer avec précision, les deux domaines ne se distinguant que par leur degré plus ou moins grand de généralité (Word and Object, MIT Press, 1960, §56, p. 275).

${ }^{8}$ L. Brunschvicg : Les progrès de la conscience européenne, Paris, PUF, 1953, t. 2, p. 530531.

${ }^{9}$ A Audiffrent, 12 février 1857, C.G., t. 8, p. 400. 
Descartes avait encore dû se contenter de reconduire sa morale provisoire. La marche ascendante, suivie dans le Cours, qui va du monde à l'homme, restaure l'unité perdue en satisfaisant simultanément des exigences tenues pour séparées. Avec la sociologie, la science pour la première fois devient humaine, entrant du même coup en rivalité avec la théologie. Le pas décisif n'est toutefois accompli que dans les conclusions générales du Cours, qui décident de confier à ce qui est encore la science finale la présidence de l'échelle encyclopédique. Désormais, la sociologie prend les commandes et une de ses fonctions sera de coordonner la marche des sciences qui la précèdent.

Ce faisant, le positiviste s'inscrit en faux contre tous ceux qui ont cru devoir opposer sciences de la nature et sciences de l'homme, relevant chacune de méthodes radicalement incompatibles : l'explication dans un cas et la compréhension dans l'autre. Les théoriciens des Geisteswissenschaften accusent leurs adversaires d'appliquer inconsidérément aux phénomènes humains des méthodes qui, si elles ont fait leur preuve dans les sciences naturelles, ne sont plus adaptées au nouvel objet qu'elles se proposent d'étudier. Le choix d'une méthode doit être déterminé par la nature de l'objet examiné; le propre des phénomènes humains étant d'être intentionnels, porteurs de sens, toute étude qui n'en tiendrait pas compte se condamnerait par avance à manquer son objet et c'est pourquoi les sciences de l'homme sont vouées à la méthode herméneutique. La formation polytechnicienne de Comte le disposait peu à se reconnaître dans une position où il n'est pas difficile de reconnaître l'expression d'un point de vue beaucoup plus classique, celui de l'exégèse et de la philologie. Il ne s'agit pas de nier que les sciences sociales soient des sciences historiques, ni qu'elles doivent se forger des méthodes nouvelles adaptées à leur objet; mais l'auteur du Cours remplace le dualisme par une extension graduelle, la classification des sciences lui permettant de reconnaître la diversité des sciences sans sacrifier pour autant leur unité.

Outre cet aspect méthodologique, la question de l'unité de la science présente un aspect pratique dont on ne saurait surestimer l'importance. L'unité de la science n'est jamais acquise et sert plutôt d'idée régulatrice. Dans les faits, la spécialisation va croissante et la cité savante se transforme chaque jour davantage en une juxtaposition de spécialistes qui répugnent à s'écarter de leur pré carré, de peur de trouver la place occupée à leur retour. Dans ces conditions, affirmer l'unité de la science, c'est réagir contre une tendance dont les effets indésirables menacent de compromettre ces mêmes progrès scientifiques qu'elle contribue à réaliser, et chercher à ramener les esprits dispersifs à la considération de l'ensemble. Une fois la part faite de ce qu'il a pu y avoir d'excessif dans cette défense de l'esprit d'ensemble, Comte se trouve en accord avec les néo positivistes dans cette idée que l'unité de la science est à réaliser presque de haute lutte. On commence en effet à découvrir que le Cercle de Vienne avait, de l'épistémologie, une conception beaucoup moins étroite que celle qui lui a longtemps été prêtée et qu'il n'ignorait pas la dimension politique de la science. Le fait est particulièrement clair dans le cas de Neurath, qui se plaçait lui aussi à un point de vue sociologique, y compris dans le fameux débat sur l'incorrigibilité des énoncés protocolaires ${ }^{10}$. Bien plus les deux positivismes s'accordent encore pour lier étroitement la question de l'unité de la science à celle de sa fonction sociale.

Le point de vue sociologique en philosophie.

${ }^{10}$ D. Zolo : Reflexive Epistemology, The philosophical Legacy of Otto Neurath, Dordrecht, Kluwer, 1989. 
Comte a voulu appeler sociologique ce point de vue philosophique. Le fait n'est pas neutre et enveloppe une idée particulière de ce qu'est la philosophie. Pourquoi cette coordination des sciences, qui transforme la science en philosophie, relève-t-elle de la sociologie ? Quoiqu'elle en dérive, la conception actuelle de cette dernière discipline ne correspond que de très loin à celle qu'en avait son fondateur et qui consacre l'universelle prépondérance du point de vue social non seulement, ce qui est assez aisément compréhensible, dans le domaine pratique mais aussi, ce qui l'est beaucoup moins, dans le domaine théorique.

La justification la plus immédiate de cette façon de voir consiste à la rapprocher de ce relativisme que Comte a toujours donné comme un des attributs les plus caractéristiques de la pensée positive. Des premiers écrits aux derniers, l'idée revient comme un leit motiv : en passant à l'état positif, l'esprit apprend à renoncer aux connaissances absolues que recherchait en vain le régime théologico-métaphysique, pour se contenter du relatif. Mais relatif est un terme lui-même relatif : relatif, soit; mais à quoi ? La doctrine présente donc différents aspects. En premier lieu "toutes nos connaissances réelles sont nécessairement relatives, d'une part au milieu en tant que susceptible d'agir sur nous, et d'une autre part à l'organisme en tant que sensible à cette action: en sorte que l'inertie de l'un ou l'insensibilité de l'autre suppriment aussitôt ce commerce continu d'où dépend toute notion effective". Comte réinterprète dans ce cadre la philosophie de Kant, à qui il reconnaît le mérite d'avoir tenté "le premier, d'échapper directement à l'absolu philosophique"; mais l'entreprise, ajoute-t-il, était condamnée à l'échec car "rien de vraiment décisif n'était possible à cet égard tant que cette évolution n'était pas convenablement étendue jusqu'aux spéculations sociales"11. Le relativisme consacre donc bien la prépondérance du point de vue social : tout doit être rapporté à l'état de la civilisation. Par exemple, la question : quelle est la meilleure forme de gouvernement possible ? doit être rejetée comme métaphysique. Prise ainsi dans l'absolu, elle n'a pas de sens et n'admet aucune réponse satisfaisante. Ce qu'il faut chercher à la place, c'est : quelle est, pour une société donnée, la forme de gouvernement la meilleure ? ce qui ne peut rien signifier d'autre que : qui lui soit la mieux adaptée. Les sympathies affichées par Comte envers le dogmatisme interdisent d'interpréter cette position, que l'on a parfois appelé "sociologisme", comme un scepticisme $^{12}$; on y verra plutôt un corollaire de cette théorie du consensus qui sert de fondement à la statique sociale et qui veut que "chacun des nombreux éléments sociaux, cessant d'être envisagé d'une manière absolue et indépendante, soit toujours exclusivement conçu comme relatif à tous les autres $^{\prime 13}$. Reposant comme elle le fait sur le principe des conditions d'existence, version positive de l'idée de cause finale, la théorie du consensus a été accusée de verser dans l'optimisme, ce contre quoi Comte s'est vigoureusement défendu, tout admettant qu'elle "tend à toujours considérer l'état social, envisagé sous tous ses divers aspects principaux, comme ayant été

${ }^{11}$ C., $58^{\text {ième }} 1$. , respectivement p. 113,111 et 112 . Ces pages contiennent un des exposés les plus explicites sur le sujet.

${ }^{12} 1844$, p. 67sqq. Le refus de l'absolu ne prémunit pas seulement contre l'arbitraire; il empêche également d'adhérer à toute forme d'atomisme logique, puisqu' "aucun fait isolé ne saurait vraiment être incorporé à la science, jusqu'à ce qu'il ait été convenablement lié à quelque autre notion" (C., $58^{\text {ième }} 1 .$, p. 94).

${ }^{13}$ C., $48^{\text {ième }} 1 .$, p. 100. 
essentiellement aussi parfait, à chaque époque, que le comportait l'âge correspondant de l'humanité"14.

Cette première approche passe toutefois sous silence un aspect capital : la façon dont la sociologie assure la coordination des sciences, la façon dont, avec elle, la science devient philosophie. Considérée sous cet angle, la prépondérance du point de vue social renvoie bien sûr au rôle social de la science, mais plus encore au contrôle de la science par la société.

Il ne sera pas nécessaire de s'arrêter longuement sur le premier aspect, car les esprits y sont naturellement sensibles. Pour la vaste majorité de nos semblables, la valeur de la science tient à ses applications. Encore qu'il faille se garder de tous les contresens qui entourent des déclarations de ce genre, le positiviste tient l'idée d'une "science pour la science" aussi peu admissible que l'idée de l'art pour l'art. La science est rarement cultivée pour elle même et, quand elle l'est, il n'est pas sûr que cet amour désintéressé mais, en un sens, égoïste de la vérité doive être donné en exemple. Ancien élève de Polytechnique, Comte raisonne en ingénieur. Ceci vaut aussi de la façon dont il approche la science sociale : la politique positive s'appuie sur une ingénierie sociale chargée de résoudre question sociale ou problèmes de société.

Afin que cette fonction sociale s'exerce dans les meilleures conditions possibles, Comte proposait d'introduire un droit de regard de la société sur les activités du savant. Comme il le notait avec satisfaction, le public "sent de plus en plus que les sciences ne sont pas exclusivement réservées pour les savants, mais qu'elles existent surtout pour lui-même" ${ }^{15}$. Les bienfaits de la science n'ont rien d'automatique. Livré à lui-même, le savant risque de ne considérer que ce qui relève de sa spécialité, et d'oublier la finalité sociale de son travail. D'où la nécessité d'une instance chargée d'arbitrer les querelles disciplinaires, et de faire que les différentes disciplines oeuvrent bien dans la même direction; et puisqu'il ne peut s'agir que du service de l'Humanité ${ }^{16}$, c'est bien l'affaire du sociologue. Contrôle du sociologue, ou du public en général ? Cette dernière possibilité s'accorde à première vue assez mal avec une des idées maîtresses de la politique positive, qui présente l'anarchie intellectuelle comme le pire des maux et condamne donc sans appel la prétention de la raison individuelle à juger de tout et à faire bon marché des compétences. Quoiqu'il en soit de cette tension, la politique positive comprend encore une théorie élaborée de la raison publique. Les interventions de celles-ci s'étendent bien au delà du seul domaine politique et c'est avant tout à former cette opinion éclairée qu'est destinée l'éducation universelle ${ }^{17}$. - Cette confiance dans le jugement populaire se fonde sur une certaine idée des rapports de la science et du bon sens. Comte est un théoricien de la continuité et l'on chercherait en vain chez lui l'équivalent de notions comme celles de rupture épistémologique ou d'incommensurabilité. La science est le prolongement du bon sens. Aucune frontière infranchissable ne sépare le point de vue de l'homme de la rue et celui du savant. Un prolétaire est parfaitement en mesure de comprendre dans ses grands traits le système du monde, ce qui suppose que, de son côté, le savant sache quand il le faut

${ }^{14}$ C., $48^{\text {ième }} 1$, p. 139 ; cf. p. 110.

${ }^{15} 1844$, p. 197; quelques lignes plus bas, Comte parle d' "un vaste tribunal spontané, aussi impartial qu'irrécusable, formé de la masse des hommes sensés, devant lequel viendront s'éteindre irrécusablement beaucoup de fausses opinions scientifiques".

16 "L'univers doit être étudié non pour lui-même, mais pour l'homme, ou plutôt pour l'humanité" 1848, p. 76.

17 Mary Pickering : Auguste Comte et la sphère publique de Jurgen Habermas, dans le volume d'Actes cité n. 2, p. 229-237. 
renoncer à son jargon. Comme le dit excellemment Annie Petit, "le bon sens universel est à la fois pour Comte une source de l'esprit positif et un moyen de contrôle"18.

\section{La méthode subjective.}

Quoique le sujet soit très controversé, c'est sans doute à partir du point de vue sociologique qu'il faut aborder la question de la méthode subjective qui, après 1848, vient compenser les inconvénients de la méthode objective suivie jusqu'alors, et qui a fort embarrassé les interprètes.

Les termes du problème avaient été très clairement posés dans la première page de la quarantième leçon du Cours, qui constitue indubitablement un des passages les plus remarquables de l'ouvrage. Remarquable tout d'abord par sa place puisque ce début des leçons consacrées à la biologie marque la principale césure de l'ouvrage : avec les êtres organisés, il faut changer de méthode, renoncer à l'analyse et aller désormais du tout à ses parties. Remarquable plus encore par sa teneur. Toutes nos conceptions, commence par y rappeler l'auteur, tournent autour de deux objets : l'étude de l'homme et celle du monde; il en résulte deux méthodes possibles, objective ou subjective, selon que l'on va du monde à l'homme ou de l'homme au monde. "Quoique, poursuit le texte, parvenue à sa pleine maturité, la vraie philosophie doive inévitablement tendre à concilier, dans leur ensemble, ces deux méthodes antagonistes, leur contraste constitue néanmoins le germe réel de la différence élémentaire entre les deux grandes voies philosophiques, l'une théologique, l'autre positive, que notre intelligence a dû suivre successivement"19.

L'homme à sa naissance ignorant tout du monde qui l'entoure, il est possible à la fois de reconnaître le bien fondé du "connais-toi toi-même" et d'affirmer que l'homme à ses débuts ne connaît essentiellement que lui-même. L'esprit était donc condamné à commencer par se projeter sur le monde et à l'interpréter à son image. Dans la mesure toutefois où la connaissance est connaissance de l'objet, son progrès passait par l'abandon d'un tel anthropomorphisme et l'adoption d'une méthode opposée à la précédente, où ce serait plutôt l'objet qui vient laisser son empreinte sur l'esprit. Mais quand cette marche du monde à l'homme a atteint son terme, que la méthode objective s'est étendue jusqu'aux phénomènes les plus authentiquement humains, la condamnation lancée contre la méthode subjective n'a plus lieu d'être et la conciliation annoncée au début de la quarantième leçon devient réalité. Un examen plus attentif montre en effet que le vice fondamental de la méthode initiale "ne consistait point dans son caractère subjectif mais dans sa nature absolue" ${ }^{\prime 20}$. La subjectivité en tant que telle est mise hors de cause et, pour que tout revienne dans l'ordre, il suffit que la méthode subjective renonce aux causes pour s'en tenir aux lois, ou si l'on préfère "qu'elle devienne sociologique au lieu de rester théologique"21. c'est donc bien l'existence du point de vue sociologique qui légitime l'usage fait de la méthode subjective dans le Système ${ }^{22}$.

A l'instar de tout ce qui relève de la seconde carrière de Comte, la méthode subjective a été, dans l'ensemble, sévèrement jugée, à commencer par les

\footnotetext{
${ }^{18}$ Note dans 1844, p. 197-198.

${ }^{19}$ C., $40^{\text {ième }} 1 .$, p. 666.

${ }^{20} \mathrm{~S}$, t. 1, p. 581 .

${ }^{21}$ S, t. 1, p. 446.

22 "La fondation de la sociologie permet à la méthode subjective d'acquérir enfin la positivité qui lui manquait, en nous plaçant irrévocablement au point de vue vraiment universel" ( $S, 1$, p. 446); cf. encore 1848 p. 75.
} 
disciples de la première heure, comme Littré et Mill. De fait, il n'est pas interdit de la tenir pour une des principales responsables des bizarreries, - pour ne pas dire plus - , qui abondent dans le Système ou dans la Synthèse subjective, et qui ont tant contribué à en discréditer l'auteur. Elle peut encore être accusée d'être l'expression d'un utilitarisme étroit, quand ce n'est pas de vouloir faire régner dans le monde savant un ordre moral qui n'y a pas sa place. En soumettant toutes les sciences au point de vue humain, elle tendrait à freiner indûment le progrès scientifique. Qui peut en effet mesurer les conséquences pour l'humanité d'une théorie mathématique, ou de la découverte d'un nouvel élément chimique?

Le positivisme n'est pas sans réponse devant ce genre d'objections; il peut par exemple présenter sa position comme la reprise de thèmes kantiens ${ }^{23}$. La méthode subjective présente quelques caractères propres qui ne sont pas tout à fait dénués de valeur, comme le montrent certaines des applications qui en ont été faites à la biologie. Puisqu'il s'agit de parcourir dans l'ordre descendant l'échelle encyclopédique, c'est en effet au premier chef sur la science qui l'y précède immédiatement que s'est exercé l'influence de la sociologie. Le cas le plus célèbre est sans conteste le tableau cérébral, qui fait de Comte un précurseur des neurosciences ${ }^{24}$. Moins connue, moins élaborée, la "théorie synthétique des maladies" exposées dans les lettres au Docteur Audiffrent n'est pas moins significative. Pour la tourner en ridicule, il suffit de rappeler que les épidémies de choléra qui ont suivi les révolutions de 1830 et de 1848 y sont attribuées à un essor insuffisant de l'altruisme. Du moins a-t-elle le mérite d'insister sur la part considérable et souvent négligée qui revient au social dans tout ce qui touche à la santé et particulièrement à l'hygiène ${ }^{25}$.

De surcroît, la méthode objective conserve une valeur irremplaçable. Le positivisme complet, c'est-à-dire religieux, se réserve le droit d'user alternativement des deux méthodes et ne remet pas en cause le principe qui veut que les pensées doivent être systématisées avant les sentiments. La prépondérance continue du coeur proclamée après 1845 est en effet de l'ordre du devoir être autant que de l'être. A lui seul, le coeur est impuissant à réaliser cette unité dans laquelle Comte voit l'état religieux par excellence; il a besoin pour cela du concours de l'intelligence, toute chétive qu'elle est, - ce que le Système résumera dans la formule lapidaire : régler le dedans par le dehors ${ }^{26}$.

23 "Comme Kant l'a bien senti, chacune de nos opinions est à la fois subjective et objective, notre esprit y étant à la fois actif et passif" ( $S$, t. 1, p. 712).

${ }^{24}$ Cf. L. Clauzade : Le statut épistémologique du tableau cérébral et la notion de type chez Auguste Comte, dans : M. Bourdeau et Fr. Chazel (éds) : Auguste Comte et l'idée de sciences de l'homme, Paris, L'Harmattan, 2002, p. 111-130; ainsi que B. Andrieux : Le laboratoire $d u$ cerveau psychologique, Paris, CNRS Editions, 2003, chap. 3. On en rapprochera également une définition sociologique du cerveau, comme organe de l'action des morts sur les vivants, proposée dans les lettres sur la maladie adressées au Docteur Audiffrent.

Comme exemple des bizarreries, ou, pour parler avec leur auteur, des "utopies positives" mentionnées un peu plus haut, on rappellera que Comte, fort de ce que "les vaches norvégiennes digèrent très bien le poisson $\mathrm{sec}^{\prime \prime}$, avait proposé de transformer les herbivores en carnassiers ( $S$, respectivement t. 4, p. 275 et t. 1, p. 604).

${ }^{25}$ Voir par exemple J.-F. Braunstein : Auguste Comte et la philosophie de la médecine, p. 159-176 du volume publié sous la direction d'A. Petit cité n. 2.

${ }^{26}$ La formule développée avait été donnée dès 1848 : “Le principal artifice du perfectionnement humain consiste, au contraire, à diminuer l'indécision, l'inconséquence et la divergence de nos desseins quelconques, en rattachant à des motifs extérieurs celles de nos habitudes intellectuelles, morales et pratiques, qui 
C'est ainsi en particulier que, l'ontogenèse récapitulant la phylogenèse, le programme des études des futures écoles positivistes restera fidèle à la méthode objective et invitera l'élève à s'élever peu à peu du monde à l'homme.

Quoi que l'on puisse penser de la méthode subjective, un fait peut être tenu pour établi. L'apport de Comte à l'histoire de la pensée tient autant aux diverses thèses auxquelles son nom reste associé qu'à l'introduction d'une nouvelle façon de philosopher. Il a choisi de philosopher d'un point de vue sociologique, et l'originalité de cette position n'est pas étrangère aux difficultés que cette oeuvre oppose à ses lecteurs. Si le programme assez élaboré de sociologie des sciences qu'il a été ainsi amené à présenter est passé le plus souvent inaperçu, c'est qu'il n'a pu être explicité qu'une fois la sociologie déjà constituée, c'est-àdire dans les conclusions générales du Cours, et qu'ensuite la promesse faite de rédiger le traité de politique positive ne lui a pas laissé le temps d'en entreprendre la réalisation. Le programme comprend deux volets : en tant qu'il représente le seul point de vue universel, le sociologue est chargé de coordonner l'ensemble des activités scientifiques (nous dirions aujourd'hui : de fixer les grandes lignes de la politique scientifique), notamment de trancher les querelles disciplinaires et, de façon plus générale, de lutter contre les effets pervers de la spécialisation en faisant valoir les droits de l'esprit d'ensemble. Mais, lorsqu'il agit de la sorte, le sociologue n'est en quelque sorte que le porte parole de l'opinion publique éclairée et Comte envisageait donc également un contrôle social de la science.

Le fondateur de la sociologie avait assigné à celle-ci une double fonction : être un science parmi les autres, ayant pour objet les faits sociaux; veiller au développement harmonieux des diverses sciences existantes. Les sociologues qui sont venus après Comte, estimant que la science des faits sociaux était encore dans l'enfance, ont concentré tous leurs efforts sur le développement de leurs discipline, au détriment de la seconde des tâches. Avec la reconnaissance institutionnelle de la sociologie, la situation a changé et il existe maintenant une sociologie des sciences, mais dont le rapport avec ce qui précède apparaît encore assez lointain. Il en va à peu près de même chez les épistémologues qui, après avoir longtemps manifesté une préférence quasi exclusive pour le contexte $\mathrm{du}$ justification, au détriment du contexte de découverte, ont redécouvert il y a quelque temps que la science était aussi une activité sociale. A cet égard, le nom de post-positivisme apparaît assez trompeur dans la mesure où les nouvelles tendances par là désignées ont pour caractéristique de réactiver des thèmes positivistes un temps oubliés ${ }^{27}$.

émanèrent d'abord de sources purement intérieures. Car tous les liens mutuels de nos diverses tendances sont incapables d'en assurer la fixité, jusqu'à ce qu'ils trouvent au dehors un point d'appui inaccessible à nos variations spontanées" (68).

${ }^{27}$ Voir par exemple B. Bensaude : La science contre l'opinion, Les Empêcheurs de penser en rond, Paris, 2003, p. 11. 